\title{
Iridium-Catalyzed Asymmetric Hydrogenation of (2H-chromen-3-yl)methanols
}

\author{
LIU Qibin, ZHOU Yonggui* \\ Dalian Institute of Chemical Physics, Chinese Academy of Sciences, Dalian 116023, Liaoning, China
}

\begin{abstract}
Trans-cyclohexane-backbone iridium complexes with different axial chirality were synthesized from $(1 R, 2 S)$-2-(pyridin-2-yl) cyclohexanol, and their application in asymmetric hydrogenation of $(2 H$-chromen-3-yl)methanols was investigated. When the hydrogenation was carried out using $1 \% \mathbf{I r}-\mathbf{8}$ as the catalyst at a hydrogen pressure of $5 \mathrm{MPa}$ in dichloromethane for $16 \mathrm{~h}$ at room temperature, excellent activities and up to $94 \%$ ee were obtained.
\end{abstract}

Key Words: (2H-chromen-3-yl)methanol; asymmetric hydrogenation; iridium catalyst

The importance of the chromane skeleton in the area of biologically active compounds is well recognized. For example, 3-hydroxymethylchromanes are important intermediates in the synthesis of $\alpha$-adrenergic blocking agents and nootropic drugs [1]. However, the preparation of optically active 3-hydroxymethylchromanes was reported in only a few examples. In 1996, Zecchi's group [2] used a molecular [3+2] cycloaddition strategy to get a series of chiral 3-hydroxymethylchromanes. Subsequently, in 2003, Hanselmann's group [3] developed Lewis acid-catalyzed [3+2] cycloaddition to synthesize 3-hydroxymethylchromane.

Through a retrosynthetic analysis (Scheme 1), the simplest method to prepare chiral 3-hydroxymethylchromanes (1) is the asymmetric hydrogenation of ( $2 H$-chromen-3-yl)methanols (2). As an extension of our work on the asymmetric hydrogenation of unfunctionalized olefins [4,5], we report here a method to synthesize chiral 3-hydroxymethylchromanes by iridium-catalyzed asymmetric hydrogenation [6].

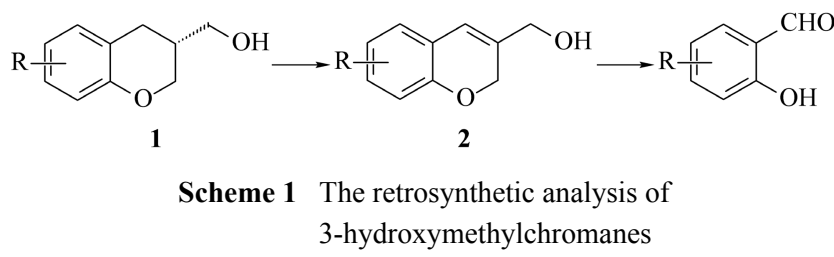

(2H-chromen-3-yl)methanols can be conveniently synthesized from substituted salicylaldehydes according to a method in the literature [7]. Using (2H-chromen-3-yl)methanol as a model substance, we evaluated five different classes of iridium complex catalysts (Scheme 2): cyclohexane-backbone Ir-1 and Ir-2 [4] and biocyclic Ir-3 [5] developed by us, and PHOX (phosphinooxazoline)-derivatized Ir-4 [8] and ThrePHOX-derivatized Ir-5 [9] developed by Pfaltz's group.
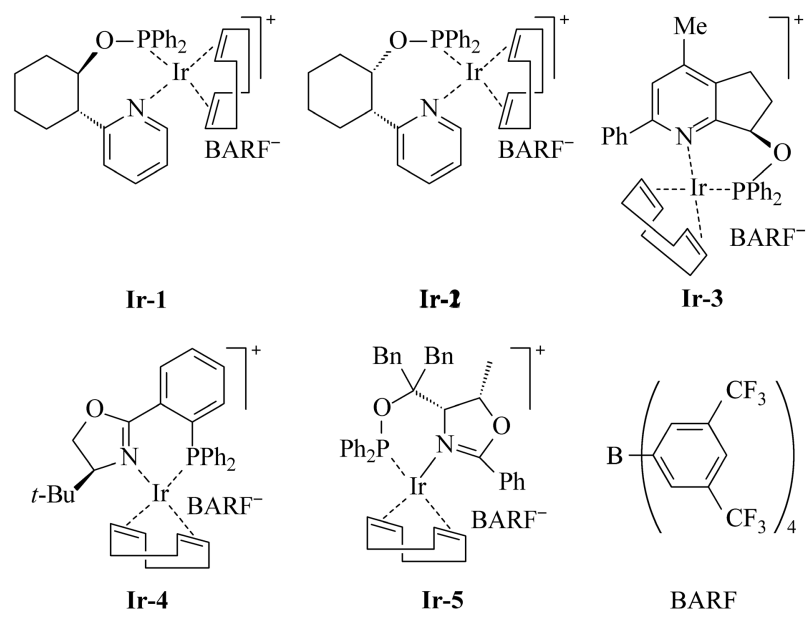

Ir-2
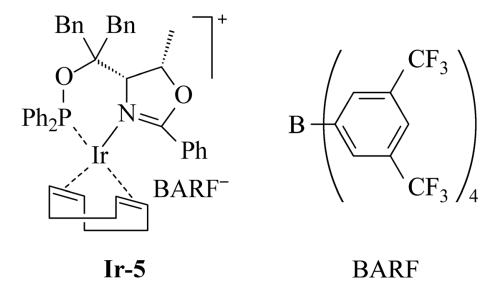

Scheme 2 Iridium complexes used in asymmetric hydrogenation of (2H-chromen-3-yl)methanol

Received date: $2007-06-19$.

* Corresponding author. Tel/Fax:+86-411-84379220; E-mail: ygzhou@dicp.ac.cn

Foundation item: Supported by the National Natural Science Foundation of China (20672112).

Copyright (C) 2007, Dalian Institute of Chemical Physics, Chinese Academy of Sciences. Published by Elsevier BV. All rights reserved. 
The hydrogenation was carried out using $1 \%$ (molar fraction) catalyst and a hydrogen pressure of $5 \mathrm{MPa}$ in dichloromethane. As shown in Table 1, only Ir-1, Ir-2, and Ir-4 gave full conversion at room temperature after $16 \mathrm{~h}$, and $\mathbf{I r - 1}$ was the most promising catalyst. In comparison to Ir-1, Ir-2 showed a slightly lower enantioselectivity $(75 \%$ ee in entry 2 vs $81 \%$ ee in entry 1 ). However, the enantioselectivity of the product using catalyst Ir-4 was much lower and only 51\% ee was obtained (entry 4). Very unfortunately, the low activity catalysts Ir-3 and Ir-5 gave poor enantioselectivities (52\% ee in entry 3 and $34 \%$ ee in entry 5). After a careful comparison, the cyclohexane-backbone Ir-1 series was chosen to further improve the enantioselectivity of the reaction.

Table 1 Iridium-catalyzed asymmetric hydrogenation of (2H-chromen-3-yl)methanol

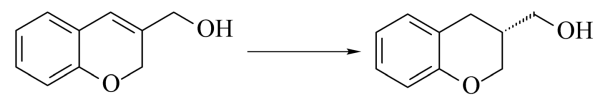

2a 1a

\begin{tabular}{cccc}
\hline Entry & Complex & Conversion $^{\mathrm{a}}(\%)$ & $\mathrm{ee}^{\mathrm{b}}(\%)$ \\
\hline 1 & Ir-1 & $>99$ & $81(R)$ \\
2 & Ir-2 & $>99$ & $75(R)$ \\
3 & Ir-3 & 20 & $52(R)$ \\
4 & Ir-4 & $>99$ & $51(S)$ \\
5 & Ir-5 & 60 & $34(R)$ \\
6 & Ir-6 & $>99$ & $89(R)$ \\
7 & Ir-7 & $>99$ & $90(R)$ \\
8 & Ir-8 & $>99$ & $94(R)$ \\
\hline
\end{tabular}

Reaction conditions: substrate $0.25 \mathrm{mmol}$, complex $0.0025 \mathrm{mmol}$, dichloromethane $2 \mathrm{ml}$, hydrogen pressure $5 \mathrm{MPa}$, room temperature, $16 \mathrm{~h}$. ${ }^{\mathrm{a}}$ Determined by ${ }^{1} \mathrm{H}$ NMR.

${ }^{\mathrm{b}}$ Determined by chiral HPLC (OJ-H).

Considering the accessibility of materials, we decided to alter the substituting group of the phosphorus atom in the catalyst from diphenyl to the BINOL (1,1'-bi-2-naphthol) derivative. Following the literature procedure [4], enantiopure $(1 R, 2 S)-\mathbf{3}$ was deprotonated with $n$-BuLi and then treated with BINOL-derivatized chlorophosphine to afford the desired $N, P$-ligands after column separation. Subsequently, these ligands were reacted with $[\operatorname{Ir}(\mathrm{COD}) \mathrm{Cl}]_{2}$ and then exchanged with NaBARF (tetrakis[3,5-bis(trifluoromethyl)phenyl]borate sodium) to afford iridium complex catalysts Ir-6 and Ir-7 (Scheme 3). Very interestingly, both $(R)$ - and $(S)$-BINOLphosphite derivatized catalysts gave full conversion and similar enantioselectivities (89\% ee in entry 6 and $90 \%$ ee in entry 7), which were superior to Ir-1. These results inspired us to synthesize a more sterically hindered BINOL-derivatized catalyst. Therefore, 3,3'-diphenyl-(S)-BINOL-phosphite was linked to obtain catalyst Ir-8 (Scheme 3). To our delight, catalyst Ir-8 also gave full conversion and a higher enantioselectivity $(94 \%$ ee in entry 8$)$.

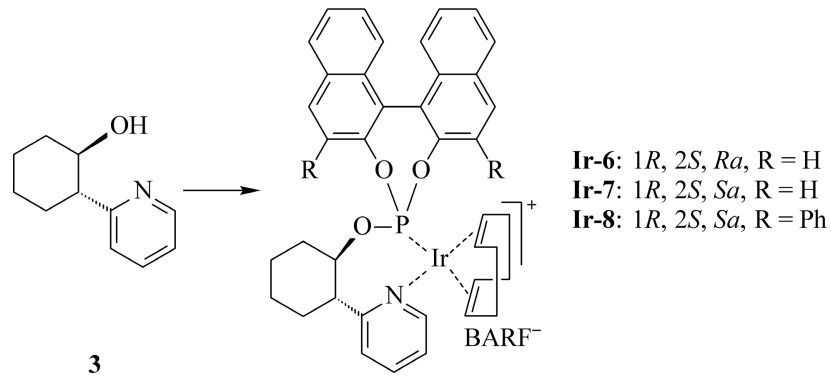

Scheme 3 Synthetic procedure for the iridium complex catalysts (The synthesis conditions are the same as in Ref. [4].)

Under the above optimized conditions, the enantioselective hydrogenation of substituted (2H-chromen-3-yl)methanols $\mathbf{2 a - 2 h}$ was evaluated using catalyst Ir-8 (Table 2). When the OMe group was changed from the 7- to 6- or 8-position, full conversion was obtained except for the 8-substituted compound ( $98 \%$ conversion in entry 2 ). However, the ee value of the products decreased from $94 \%$ to $87 \%$ or $88 \%$, respectively. Next, different 6-position substituted groups were studied to investigate their effects on activity and enantioselectivity. It was found that when the 6-position substituted group was an alkyl, full conversion was obtained (entries 5 and 6). Among these, the substances with the methyl and more sterically hindered $t$-Bu group gave $90 \%$ ee (entry 5 ) and higher $94 \%$ ee (entry 6), respectively. When the 6-position substituted group was phenyl, full conversion and lower enantioselectivity were obtained ( $85 \%$ ee in entry 7$)$. For the $\beta$-naphthyl-1-formaldehyde-derivatized substance (2h), catalyst Ir-8 gave full conversion and $92 \%$ ee (entry 8 ).

Table 2 Ir-8-catalyzed asymmetric hydrogenation of (2H-chromen-3-yl)methanols

\begin{tabular}{ccccc}
\multicolumn{2}{c}{} & & \multicolumn{3}{c}{ Conversion $(\%)$} & ee $(\%)$ \\
\hline Entry & Substrate & $\mathrm{R}$ & $>99$ & $94(R)$ \\
\hline 1 & $\mathbf{2 a}$ & $\mathrm{H}$ & 98 & $88(R)$ \\
2 & $\mathbf{2 b}$ & $8-\mathrm{OMe}$ & $>99$ & $94(R)$ \\
3 & $\mathbf{2 c}$ & $7-\mathrm{OMe}$ & $>99$ & $87(R)$ \\
4 & $\mathbf{2 d}$ & $6-\mathrm{OMe}$ & $>99$ & $90(R)$ \\
5 & $\mathbf{2 e}$ & $6-\mathrm{Me}$ & $>99$ & $94(R)$ \\
6 & $\mathbf{2 f}$ & $6-t-\mathrm{Bu}$ & $>99$ & $85(R)$ \\
7 & $\mathbf{2 g}$ & $6-\mathrm{Ph}$ & $>99$ & $92(R)$ \\
8 & $\mathbf{2 h}$ & $5,6=-(\mathrm{CH}=\mathrm{CH})_{2}-$ &
\end{tabular}

Reaction conditions are the same as in Table 1.

In summary, iridium-catalyzed asymmetric hydrogenation of (2H-chromen-3-yl)methanols to chiral 3-hydroxymethylchromanes was performed and catalyst Ir-8 showed excellent activity and up to $94 \%$ ee. 


\section{References}

[1] Mouysset G, Payard M, Grassy G, Tronche P, Dabire H, Mouille P, Schmitt H. Eur J Med Chem, 1987, 22(6): 539

[2] Broggini G, Folcio F, Sardone N, Sonzogni M, Zecchi G. Tetrahedron: Asymmetry, 1996, 7(3): 797

[3] Hanselmann R, Zhou J C, Ma P, Confalone P N. J Org Chem, 2003, 68(22): 8739

[4] Liu Q B, Zhou Y G. Tetrahedron Lett, 2007, 48(12): 2101
[5] Liu Q B, Yu C B, Zhou Y G. Tetrahedron Lett, 2006, 47(27): 4733

[6] Cui X H, Burgess K. Chem Rev, 2005, 105(9): 3272

[7] Satoh Y, Stanton J L, Hutchison A J, Libby A H, Kowalski T J, Lee W H, White D H, Kimble E F. J Med Chem, 1993, 36(23): 3580

[8] Lightfoot A, Schnider P, Pfaltz A. Angew Chem, Int Ed, 1998, 37(20): 2897

[9] Menges F, Pfaltz A. Adv Synth Catal, 2002, 344(1): 40 